\title{
Synthesis of an enantiopure thioester as key substrate for screening the sensitivity of penicillin binding proteins to inhibitors
}

\author{
Justine F. Simon, ${ }^{\text {a* }}$ André Bouillez, ${ }^{\mathrm{b}}$ Jean-Marie Frère, ${ }^{\mathrm{c}}$ \\ André Luxen, ${ }^{\mathrm{a}}$ and Astrid Zervosen ${ }^{\mathrm{a}}$ \\ ${ }^{a}$ Cyclotron Research Center, B30, University of Liege (ULg), Sart-Tilman 4000, Belgium \\ ${ }^{b}$ Organic Synthesis Laboratory, Department of chemistry, B6a, ULg, Sart-Tilman 4000, Belgium \\ ${ }^{c}$ Center for Protein Engineering, B6a, ULg, Sart-Tilman 4000, Belgium \\ E-mail: jsimon@ulg.ac.be
}

DOI: http://dx.doi.org/10.3998/ark.5550190.p009.621

\begin{abstract}
The synthesis of the enantiopure thioester (R)-2-(2-benzamidopropanoylthio)acetic acid was developed. After the exploration of several activation methods, reaction conditions were found for the formation of the thioester bond in the presence of propylphosphonic anhydride with high enantioselectivity (ee $>99 \%$ ). The thioester activity of Penicillin Binding Proteins is helpful in research programs looking for new lead structures to overcome the problem of bacterial resistance.
\end{abstract}

Keywords: Enantiopure thioester, propylphosphonic anhydride, Penicillin Binding Proteins

\section{Introduction}

Peptidoglycan is an unique structure specific to the bacterial cell and a relevant target for antibiotics. Its architecture maintains a defined cell shape and is necessary for the survival of the cell, as a protection against mechanical damage and osmotic rupture. One of the two last steps of its biosynthesis is the reticulation of the polymer strand, (GlcNAc-MurNAc-pentapeptide) $n$, catalyzed by the enzymatic feature of DD-transpeptidase (D-alanyl-D-alanine peptidase) of the High Molecular Mass Penicillin Binding Proteins, PBPs (Scheme 1). One mechanism of the $\beta$ lactam ring antibiotics is the formation of a stable adduct with the active serine of PBPs (Scheme $1)^{1}$ leading to the inhibition of peptidoglycan reticulation and bacterial lysis. 


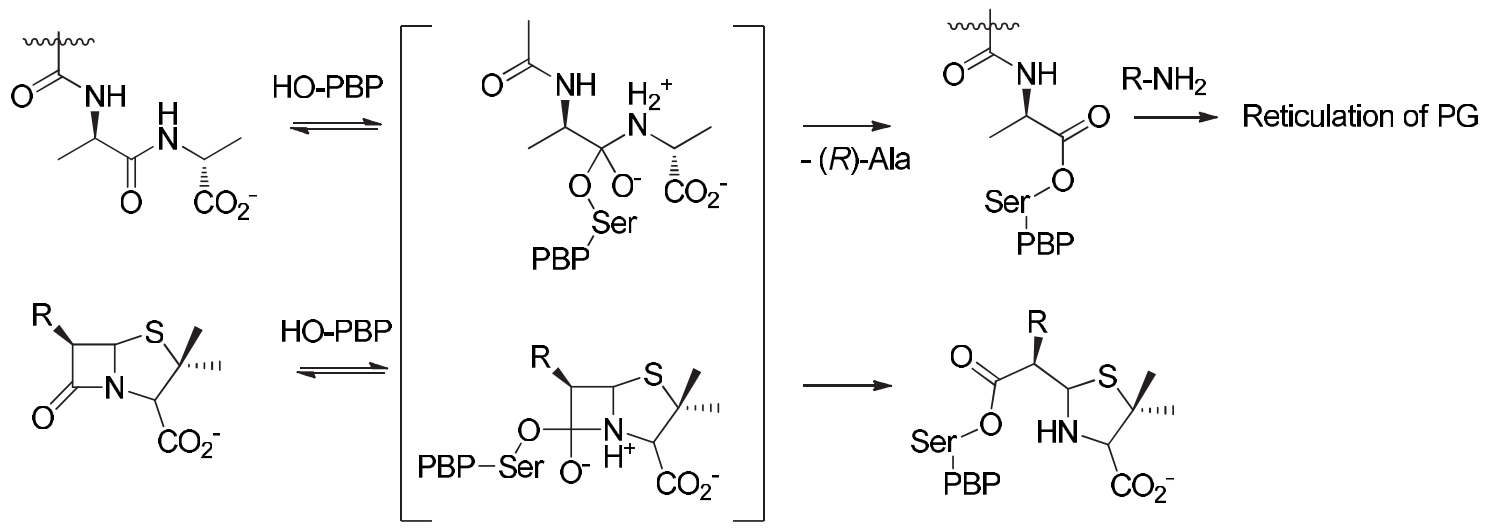

Scheme 1. Reticulation of the peptidoglycan by the action of PBP and stable covalent adduct between PBP and the $\beta$-lactam ring antibiotic.

The widespread use of $\beta$-lactam antibiotics has resulted in the production of resistant Penicillin Binding Proteins (PBPs) by some bacterial strains. Efforts by many research groups to identify new molecules as inhibitors or inactivators of resistant PBPs have been made. The development of in vitro screening assays is an important milestone in these research programs. Thioester activity of PBPs is well known ${ }^{1,2}$ and has been used to identify and characterize the inhibitors and inactivators of PBPs. ${ }^{3-13}$ Indeed, the hydrolysis of thioester, $(R)$-2-(2-benzamidopropanoylthio)acetic acid $(\boldsymbol{R}) \mathbf{- 6}$, catalyzed by PBPs leads to $(R)$-2-benzamidopropanoic acid $(\boldsymbol{R})-\mathbf{1}$ and 2mercaptoacetic acid (Scheme 2). The sensitivity of penicillin binding proteins to inhibitors can be followed by measuring the absorbance of $(R)$-2-benzamidopropanoic acid $(\boldsymbol{R})-\mathbf{1}$ at $250 \mathrm{~nm}\left(\varepsilon_{250 \mathrm{~nm}}\right.$ : $-2.200 \mathrm{M}^{-1} \mathrm{~cm}^{-1}$ ) or the sensitivity of the assay can be increased by combining 2-mercaptoacetic acid formation with thiol oxidation by 5,5'-dithiobis(2-nitrobenzoic acid) (DTNB) at $412 \mathrm{~nm}$ (E412nm: $\left.14150 \mathrm{M}^{-1} \mathrm{~cm}^{-1}\right)$.

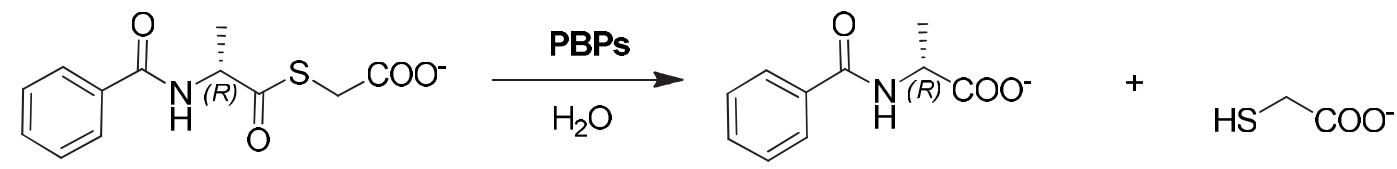

Scheme 2. Thioesterase activity of PBPs.

For enzymatic studies, different thioesters such as $(R)$-2-(2-benzamidopropanoylthio)acetic acid $(\boldsymbol{R})-6$ were reported, ${ }^{1,2,14,15}$ however a strict specificity for the $(R)$-configuration in the penultimate position was required. Syntheses of $(R)$-2-(2-benzamidopropanoylthio)acetic acid $(\boldsymbol{R})$-6 in solution starting from $(R)$-alanine or solid-supported by the use of a resin-bound $\alpha$ hydroxy ester were described in literature, ${ }^{1,14,16}$ but the enantiomeric purity was not studied. The major problem of thioester syntheses was to maintain the configuration of the asymmetric carbon 
in the penultimate position. In peptide coupling the activation step is critical, therefore methods to prevent racemization have been intensively studied. ${ }^{17}$ As shown in the literature, information on the epimerization of other thioester derivatives is available where base effects are determined. ${ }^{16}$ Here, different coupling methods for thioester synthesis were explored, evaluating their enantiomeric purity by chiral HPLC. Among the new coupling agents commercially available, propylphosphonic anhydride (T3P) was selected. It is known to reduce epimerization during peptide synthesis and we expect it could be used in thioester synthesis.

\section{Results and Discussion}

\section{Synthesis of $(R)-6$}

$(R)$-2-Benzamidopropanoic acid $(\boldsymbol{R})$-1 was synthesized from $(R)$-alanine and benzoyl chloride using a Schotten-Baumann reaction under conditions described in the literature with good yield (88\%) (i, Scheme 3). ${ }^{14}$

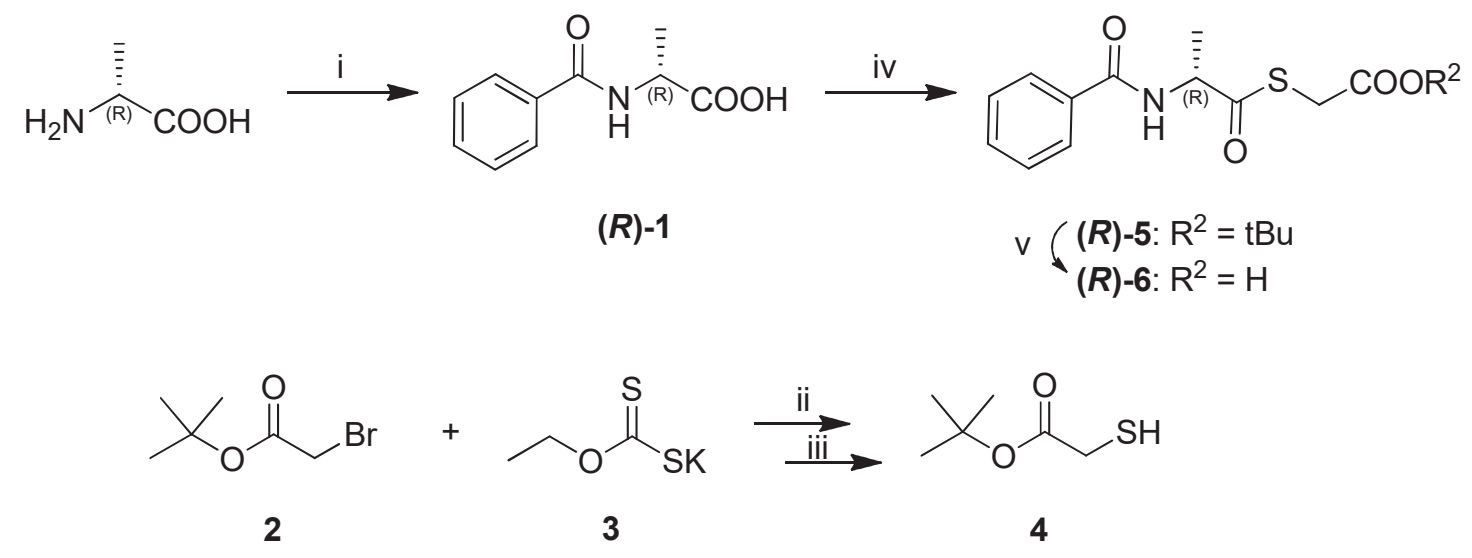

Scheme 3. Synthesis of (R)-6, i) benzoyl chloride, $\mathrm{H}_{2} \mathrm{O}, \mathrm{pH}=10,0{ }^{\circ} \mathrm{C}$; ii) ethanol, $0{ }^{\circ} \mathrm{C} \rightarrow \mathrm{rt}$, $15 \mathrm{~h}$; iii) 2 equiv ethanolamine at $0{ }^{\circ} \mathrm{C}, 2 \mathrm{~h}$ room temperature; iv) reagents as listed in Table 1 ; v) 2 equiv of TFA at $0^{\circ} \mathrm{C}, 2 \mathrm{~h}$ at $\mathrm{RT}$, water.

The second step consisted of a coupling reaction between $(R)$-2-benzamidopropanoic acid $(\boldsymbol{R})$ $\mathbf{1}$ and tert-butyl-2-mercaptoacetate 4 (iv, Scheme 3) or commercial available 2-mercaptoacetic acid. 4 was previously synthesized in two steps from potassium ethylxanthogenate 3 and tert-butyl2-bromoacetate 2 with moderate yields (53\%) as described by Kricheldorf ${ }^{18}$ (ii and iii, Scheme 3). For the coupling, three methods of activation were studied as listed in Table 1.

1.1. Activation with DCC. Llinas ${ }^{14}$ synthesized 6 from $(\boldsymbol{R})-\mathbf{1}$ and $\mathbf{4}$ using DCC activation at room temperature but no information about the enantiomeric excess (ee) was provided. We 
synthesized 6 using DCC activation under conditions described by Neises ${ }^{19}$ followed by deprotection with trifluoroacetic acid.

To determine the enantiomeric purity of the thioester, a chromatographic method was developed to distinguish the two enantiomers $(\boldsymbol{R}) \mathbf{- 6}$ and $(\boldsymbol{S})-\mathbf{6}$ by two different retention times using a Chiral HPLC (ChiralCel OD-H Daicel column: $250 \times 4.6 \mathrm{~mm}$; flow: $0.65 \mathrm{~mL} / \mathrm{min}$ ). 8/2/0.1 n-hexane/isopropanol/TFA at $30^{\circ} \mathrm{C}$ is optimal and the enantiomer $(\boldsymbol{R})-\mathbf{6}$ (Figure 1a) was first eluted after 12.5 min followed by $(\boldsymbol{S})-6$ (Figure 1c) at $15.1 \mathrm{~min}$. Entry 1 (Table 1 ) showed $50 \%$ of each isomers (Figure 1b).
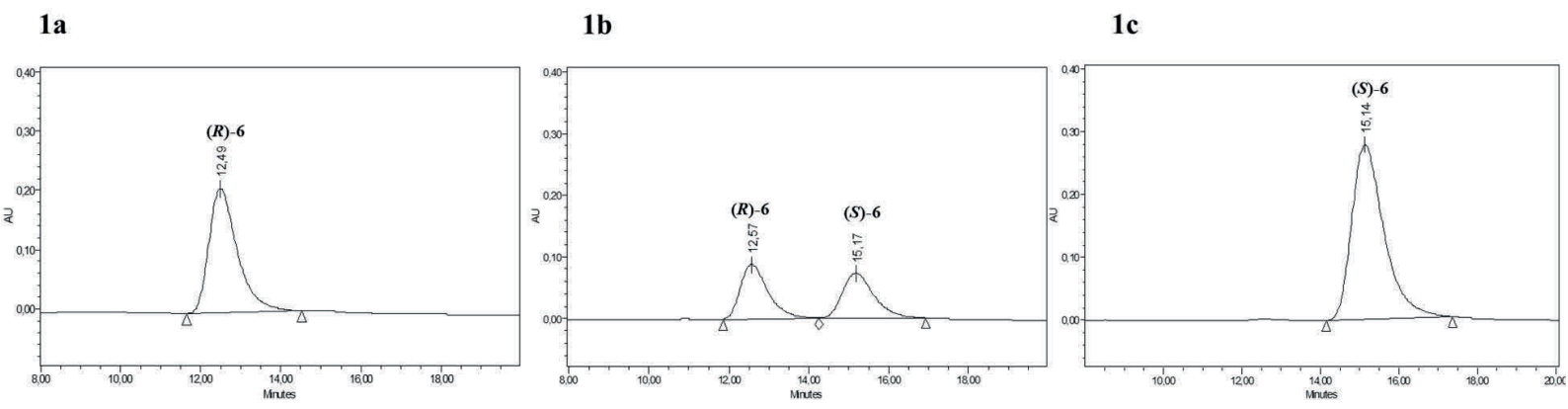

Figure 1. Chiral HPLC (228 nm) of 1a) enantiomer $(\boldsymbol{R})-\mathbf{6}, 1 \mathrm{~b})$ rac-6 and 1c) enantiomer (S)-6

1.2. Activation with ethyl chloroformate (ECF) or isobutyl chloroformate (IBCF). Adam ${ }^{2}$ and Scwyzer ${ }^{20}$ used ECF to activate carboxylic acids followed by the reaction with 2mercaptoacetic acid. We synthesized 6 under these experimental conditions and analyzed the epimerization by chiral HPLC described above. As listed in Table 1, 6 is racemic (entry 2). The nature of the base (triethylamine replaced by $N$-methylmorpholine (NMM)), the activation reagent (ECF by IBCF) and the reaction temperature were modified to improve the enantiomeric excess as listed in Table 1 (entries 3 and 4).

1.3. Activation with T3P. The activation was effected by propylphosphonic anhydride (T3P) with $(\boldsymbol{R})-\mathbf{1}$ and $\mathbf{4}$ at three different temperatures. Solvents used for the coupling were adapted to the solubility of reagents at the desired temperatures. Decrease of the temperature increased the enantiomeric excess (entries 5-7, Table 1 ). At $-78^{\circ} \mathrm{C}$, the enantiopurity of the deprotected product (R)-6 was confirmed (ee: $>99 \%$ ).

Under these conditions a scale-up was done and $1 \mathrm{~g}$ of $(\boldsymbol{R})-\mathbf{5}$ was synthesized from $(\boldsymbol{R})-\mathbf{1}$ and 4 in very moderate yields (33\%). The deprotection was done with 2 equivalents of TFA at $0{ }^{\circ} \mathrm{C}$. After stirring for $2 \mathrm{~h}$ at room temperature the reaction was quenched with water. $(\boldsymbol{R})-\mathbf{6}(0.8 \mathrm{~g})$ was isolated as a white solid in good yields $(85 \%$, ee $>99 \%)$. The NMR-data of $(\boldsymbol{R})-\mathbf{5}$ and $(\boldsymbol{R})-\mathbf{6}$ were in agreement with literature data. ${ }^{14}(S)$-2-(2-benzamidopropanoylthio)acetic acid $((S)-6)$ was synthesized in the same way and used for chiral HPLC and biological experiments. 
Table 1. The Chiral HPLC method described allowed the determination of the enantiomeric excess (ee) of deprotected $(\boldsymbol{R})-\mathbf{6}$ synthesized under different conditions

\begin{tabular}{|c|c|c|c|c|c|c|c|}
\hline Entry & Thiol derivatives & Activator & $\begin{array}{c}\mathrm{T} \\
{\left[{ }^{\circ} \mathrm{C}\right]}\end{array}$ & Base & Solvent & $\begin{array}{c}\text { Yield } 1 \rightarrow 6 \\
{[\%]}\end{array}$ & $\begin{array}{c}\text { ee } \\
{[\%]}\end{array}$ \\
\hline 1 & 4 & DCC & 20 & DMAP & DCM/DMF:4/1 & 58 & 0 \\
\hline 2 & $\begin{array}{c}\text { 2-mercaptoacetic } \\
\text { acid }\end{array}$ & $\mathrm{ECF}^{6,7}$ & -5 & $\mathrm{Et}_{3} \mathrm{~N}$ & EtOAc & 83 & 0 \\
\hline 3 & $\begin{array}{c}\text { 2-mercaptoacetic } \\
\text { acid }\end{array}$ & $\mathrm{IBCF}$ & -25 & NMM & THF & n.d. & 18 \\
\hline 4 & $\begin{array}{c}\text { 2-mercaptoacetic } \\
\text { acid }\end{array}$ & $\mathrm{IBCF}$ & -78 & $\mathrm{NMM}$ & THF & n.d. & 72 \\
\hline 5 & 4 & T3P & 0 & NMM & $\mathrm{ACN}$ & 68 & 0 \\
\hline 6 & 4 & T3P & -25 & NMM & EtOAc & 28 & 74 \\
\hline 7 & 4 & T3P & -78 & NMM & EtOAc/ACN:4/1 & 28 & $>99$ \\
\hline
\end{tabular}

\section{Biological experiments}

The use of our coupling method could be helpful in the thioester research programs looking for new lead structures to overcome the problem of bacterial resistance caused by the widespread use of $\beta$-lactam antibiotics. The activities of our thioester with PBPs from different classes ${ }^{8}$ with R39 from Actinomadura (class C) and PBP2x from a penicillin sensitive (PBP2xR6) and resistant (PBP2x5204) strain of Streptococcus pneumoniae (class B) and their use in the screening using microtiter plate are described in the Supplementary Data. Enzymatic results show the importance of the enantiopurity of $(\boldsymbol{R}) \mathbf{- 6}$ for its thioesterase activity.

\section{Conclusions}

In conclusion, we have developed a synthesis of the enantiopure thioester $(\boldsymbol{R})-\mathbf{6}$ in an overall yield of $25 \%$ from $(R)$-alanine. The described chemical synthesis can be used for the synthesis of enantiopure thioester libraries. As $\mathbf{6}$ is commercially available but its enantiomeric purity is not always reported, our chromatographic method will be useful to quantify its enantiomeric purity.

\section{Experimental Section}

General. All chemicals (including $\mathrm{T} 3 \mathrm{P} \AA$ ) and reagents were purchased from commercial suppliers. All solvents used were HPLC grade. The HPLC chain consists of a pump (Waters 600) 
and an UV detector (PDA Waters 996). Analytical HPLC analyses were performed on a column XTerra RP18 $(150 \times 4.6 \mathrm{~mm}, 3.5 \mu \mathrm{m}$, from Waters) using gradient (Flow: $0.7 \mathrm{~mL} / \mathrm{min})$ at $30{ }^{\circ} \mathrm{C}$. The following gradient was used: solvent A: water (MilliporeQ) containing $0.1 \% \mathrm{TFA} \mathrm{v} / \mathrm{v}$ and solvent B: ACN, gradient: 0 - 30 min: $0-100 \% \mathrm{~B}, 30-35 \mathrm{~min}: 100 \% \mathrm{~B}$. The injection volume was $20 \mu \mathrm{L}$. Chiral HPLC analyses were performed on a column ChiralCel OD-H $(250 \times 4.6 \mathrm{~mm}$, from Daicel) using n-hexane/isopropanol/TFA (8/2/0.1) as solvent (Flow: $0.65 \mathrm{~mL} / \mathrm{min}$ ) at regulated $30{ }^{\circ} \mathrm{C}$. The injection volume was $20 \mu \mathrm{L}$, solid compound was first dissolved in isopropanol followed by dilution in hexane. ${ }^{1} \mathrm{H}$ NMR and ${ }^{13} \mathrm{C}$ NMR spectra were recorded at $298 \mathrm{~K}$ on a Bruker $250 \mathrm{MHz}$ or $400 \mathrm{MHz}$ spectrometers. Chemical shifts $\delta$ are given in ppm. The employed abbreviations for the multiplicities are the following ones: $\mathrm{s}=$ singlet, $\mathrm{d}=$ doublet, $\mathrm{t}=$ triplet, $\mathrm{q}=$ quadruplet, $\mathrm{m}=$ multiplet, $\mathrm{br}=$ broad. Coupling constants $J$ are given in Hz. Spectra were recorded in solutions of $\mathrm{CDCl}_{3}\left(\delta_{\mathrm{H}}\right.$ at $7.26 \mathrm{ppm}, \delta_{\mathrm{C}}$ at $\left.77.1 \mathrm{ppm}\right)$ or $\mathrm{d}_{6}-\mathrm{DMSO}\left(\delta_{\mathrm{H}}\right.$ at $2.5 \mathrm{ppm}$, $\delta_{\mathrm{C}}$ at $39.5 \mathrm{ppm}$ ) which were used as internal references. MS analyses were made on a TSQ 7000 Thermoquest Finnigan mass spectrometer equipped with an electrospray source. The co-solvent (injected in $200 \mu \mathrm{L} / \mathrm{min}$ ) is a mixture $\mathrm{H}_{2} \mathrm{O} / \mathrm{ACN}(50 \%$ ) containing $0.1 \% \mathrm{AcOH} v / \mathrm{v}$. Melting points were determined using a melting point apparatus SMP3 from Stuart Scientific. Enzymatic buffers: buffer A: $10 \mathrm{mM}$ sodium phosphate $\mathrm{pH} 7.2$ with $100 \mathrm{mM} \mathrm{NaCl}$, buffer B: $10 \mathrm{mM}$ sodium phosphate $\mathrm{pH}$ 7.0. PBP2xR6 and PBP2x5204 from Streptococcus pneumoniae (class B) were prepared as described by Carapito. ${ }^{28} \mathrm{R} 39$ from Actinomadura (class C) was prepared and purified as described by Granier. ${ }^{29}$

(R)-2-benzamidopropanoic acid $((\boldsymbol{R})$-1). Synthesis was performed with benzoyl chloride (14.05 g, $0.1 \mathrm{~mol})$ and $(R)$-alanine $(0.11 \mathrm{~mol})$ as described by Llinas. ${ }^{14}(\boldsymbol{R})-\mathbf{1}$ was obtained as a white solid $(17.2 \mathrm{~g}, 88 \%), \mathrm{mp} 148.1-153.0{ }^{\circ} \mathrm{C}\left(\mathrm{lit},{ }^{30} 147-148{ }^{\circ} \mathrm{C}\right)$ after adjusting the $\mathrm{pH}$ of the solution to 2.5 by addition of $2 \mathrm{~N} \mathrm{HCl}$. TLC : (Si60, petroleum spirit/ethyl acetate/acetic acid: 50/50/0.1 $(\mathrm{v} / \mathrm{v} / \mathrm{v}), R_{f}$. 0.74). HPLC: $R_{t}: 14 \mathrm{~min}$. NMR spectra: $\delta_{\mathrm{H}}\left(400 \mathrm{MHz} ; \mathrm{d}_{6}-\mathrm{DMSO}\right) 1.41(3 \mathrm{H}, \mathrm{d}, J 7.3$, $\left.\mathrm{CH}_{3 \mathrm{Me}}\right), 4.44\left(1 \mathrm{H}, \mathrm{d}-\mathrm{q}, J_{1} 7.2, J_{2} 7.3, \mathrm{CH}_{\alpha}\right), 7.50(3 \mathrm{H}, \mathrm{m}, \mathrm{CH} \mathrm{Ph}), 7.90(2 \mathrm{H}, \mathrm{m}, \mathrm{CHPh}), 8.66(1 \mathrm{H}, \mathrm{d}$, $J$ 7.2, NH), $12.54\left(1 \mathrm{H}\right.$, br s, OH); $\delta_{C}\left(101 \mathrm{MHz}, \mathrm{d}_{6}-\mathrm{DMSO}\right) 17.35\left(\mathrm{CH}_{3}\right), 48.61(\mathrm{CH}), 127.88$ $\left(\mathrm{CH}_{\mathrm{Ph}}\right), 128.68\left(\mathrm{CH}_{\mathrm{Ph}}\right), 131.79\left(\mathrm{CH}_{\mathrm{Ph}}\right), 134.40\left(\mathrm{C}_{\mathrm{Ph}}\right), 166.62\left(\mathrm{CO}_{\mathrm{Ph}}\right), 174.69(\mathrm{COOH})$, were in agreement with Llinas. ${ }^{14}[\alpha]_{D}^{25}-33^{\circ}\left(c 0.2, \mathrm{NaOH}_{\mathrm{aq}}\right)\left(\right.$ lit. $^{30}[\alpha]_{D}^{25}-36^{\circ},\left(c 0.19, \mathrm{NaOH}_{\mathrm{aq}}\right)$. MS (ESI, positive ion) $m / z: 194[\mathrm{M}+\mathrm{H}]^{+}$.

tert-Butyl 2-mercaptoacetate (4). Potassium $O$-ethyldithiocarbonate (3) (14.27 g, $0.089 \mathrm{~mol}$ ) was dissolved in $50 \mathrm{~mL}$ of absolute ethanol. $t$-Butyl bromoacetate (2) $(12.89 \mathrm{~mL}, 0.089 \mathrm{~mol})$ was slowly added at $0{ }^{\circ} \mathrm{C}$. After $15 \mathrm{~h}$ at room temperature ethanol was removed by evaporation and chloroform $(50 \mathrm{~mL})$ and water $(50 \mathrm{~mL})$ were added. The product was extracted with chloroform $(3 \times 50 \mathrm{~mL})$. The organic phase was washed with an aqueous solution of $10 \% \mathrm{NaHCO}_{3}$, citric acid $(10 \%)$ and brine. The organic phase was dried with $\mathrm{MgSO}_{4}$. The solvent was removed. Under $\mathrm{N}_{2}$ atmosphere 2 equiv. of ethanolamine $(0.132 \mathrm{~mol}, 8 \mathrm{~mL})$ were added slowly at $0{ }^{\circ} \mathrm{C}$. After $2 \mathrm{~h}$ at room temperature the product was purified as described by Kricheldorf ${ }^{18}$. Ester 4 was obtained as a yellow oil (16.7 g, 53\%). TLC: (Si60, petroleum spirit/ethyl acetate/formic acid: 50/50/0.1, $R_{f}$. 0.4). ${ }^{1} \mathrm{H}$ NMR data $\delta_{H}\left(400 \mathrm{MHz} ; \mathrm{CDCl}_{3}\right) 1.48(9 \mathrm{H}, \mathrm{s}, t-\mathrm{Bu}), 1.94(1 \mathrm{H}, \mathrm{t}, J$ 8.1, SH), $3.17(2 \mathrm{H}, \mathrm{d}$, 
$J$ 8.1, $\left.\mathrm{CH}_{2}\right)$ were in agreement with Kricheldorf; ${ }^{18}{ }^{13} \mathrm{C}$ NMR data $\left(101 \mathrm{MHz} ; \mathrm{CDCl}_{3}\right) \delta_{C} 27.78$ $\left(\mathrm{CH}_{2}\right), 27.9\left(\mathrm{CH}_{3}\right), 81.80\left(\mathrm{CCH}_{3}\right), 169.93(\mathrm{CO})$ were new.

tert-Butyl (R)-2-(2-benzamidopropanoylthio)acetate ((R)-5). At $-78{ }^{\circ} \mathrm{C}$ under $\mathrm{N}_{2}$ atmosphere, $(\boldsymbol{R})-1$ (1.93 g, $0.01 \mathrm{~mol})$ was dissolved in $100 \mathrm{~mL}$ of ethyl acetate/acetonitrile solution: $4 / 1$ then ester $4(1.48 \mathrm{~g}, 0.01 \mathrm{~mol})$ was added. After the slow addition of NMM $(3.57 \mathrm{~mL}, 0.012 \mathrm{~mol})$ the reaction was started by addition of a solution of T3P cooled to $-78{ }^{\circ} \mathrm{C}(3.57 \mathrm{~mL}, 0.012 \mathrm{~mol}, 5 \%$ T3P in ethyl acetate/acetonitrile: 4/1). The reaction was followed by TLC (Si60, petroleum spirit/ethyl acetate/formic acid: 50/50/0.5, $\mathrm{R}_{\mathrm{f}}$ : 0.8) and was finished after 5 hours. After the addition of acetic acid in ethyl acetate $(0.05 \mathrm{~mol}$ in $50 \mathrm{~mL})$ the organic phase was separated and washed successively with water, sodium phosphate buffer $\mathrm{pH} 8$, citric acid (10\%), water and brine. The organic phase was dried with $\mathrm{Na}_{2} \mathrm{SO}_{4}$. The product was purified by column chromatography (silica, ethyl ether/ethyl acetate/formic acid: 80/20/0.1). $1.06 \mathrm{~g}$ (yield: 33\%) of (R)-5 a white solid was separated. The NMR-data $\delta_{H}\left(400 \mathrm{MHz}\right.$; d6-DMSO) $1.39\left(9 \mathrm{H}, \mathrm{s}, \mathrm{CH}_{3 t-\mathrm{Bu}}\right), 1.42(3 \mathrm{H}, \mathrm{d}, J 7.3$, $\left.\mathrm{CH}_{3 \mathrm{Me}}\right), 3.61\left(2 \mathrm{H}, \mathrm{s}, \mathrm{CH}_{2}\right), 4.67\left(1 \mathrm{H}, \mathrm{d}-\mathrm{q}, J_{1} 7.2, J_{2} 7.3, \mathrm{CH \alpha}\right), 7.53\left(3 \mathrm{H}, \mathrm{m}, \mathrm{CH}_{\mathrm{Ph}}\right), 7.92(2 \mathrm{H}, \mathrm{m}$, $\left.\mathrm{CH}_{\mathrm{Ph}}\right), 9.08(1 \mathrm{H}, \mathrm{d}, J 7.2, \mathrm{NH})$ and $\delta_{C}\left(101 \mathrm{MHz}, \mathrm{d}_{6}-\mathrm{DMSO}\right) 17.41\left(\mathrm{CH}_{3 \mathrm{Me}}\right), 28.02\left(\mathrm{CH}_{3 t-\mathrm{Bu}}\right), 32.10$ $(\mathrm{CH}), 55.63\left(\mathrm{CH}_{2}\right), 81.69\left(\mathrm{CCH}_{3 t-\mathrm{Bu}}\right), 127.99\left(\mathrm{CH}_{\mathrm{Ph}}\right), 128.81\left(\mathrm{CH}_{\mathrm{Ph}}\right), 132.12\left(\mathrm{CH}_{\mathrm{Ph}}\right), 134.07\left(\mathrm{C}_{\mathrm{Ph}}\right)$, $167.17\left(\mathrm{CO}_{\mathrm{Ph}}\right), 167.84(\mathrm{COO}), 201.62(\mathrm{COS})$ were in agreement with data of literature. ${ }^{14} \mathrm{MS}$ (ESI, positive ion) $\mathrm{m} / \mathrm{z}: 346[\mathrm{M}+\mathrm{Na}]^{+}$.

$(\boldsymbol{R})$-2-(2-Benzamidopropanoylthio)acetic acid $((\boldsymbol{R})-\mathbf{6})$. One equiv. of $(\boldsymbol{R})-\mathbf{5}(1.06 \mathrm{~g}, 0.003 \mathrm{~mol})$ was mixed with 2 equiv. of TFA at $0{ }^{\circ} \mathrm{C}$. After $2 \mathrm{~h}$ at $20{ }^{\circ} \mathrm{C}$, reaction was stopped with water. $(\boldsymbol{R})$ 6 was extracted with ethyl acetate. Subsequently, the organic phase was washed with a phosphate buffer at $\mathrm{pH}$ 8. The aqueous phase was acidified with citric acid (10\%) until the $\mathrm{pH}$ was 3.5 and the product was extracted with ethyl acetate and washed with brine and water. The acid $(\boldsymbol{R})-\mathbf{6}$ was isolated as a white solid $(0.8 \mathrm{~g}, 85 \%), \mathrm{mp} 141.8-142.9{ }^{\circ} \mathrm{C}$. The NMR-data: $\delta_{H}\left(400 \mathrm{MHz}\right.$; $\mathrm{d}_{6-}$ DMSO) 1.41 (3H, d, J 7.3, $\left.\mathrm{CH}_{3 \mathrm{Me}}\right), 3.64\left(2 \mathrm{H}, \mathrm{s}, \mathrm{CH}_{2}\right), 4.70\left(1 \mathrm{H}, \mathrm{d}-\mathrm{q}, J_{1} 7.2, J_{2} 7.3, \mathrm{CH}_{\alpha}\right), 7.55$ $(3 \mathrm{H}, \mathrm{m}, \mathrm{CH} \mathrm{Ph}), 7.93\left(2 \mathrm{H}, \mathrm{m}, \mathrm{CH}_{\mathrm{Ph}}\right), 9.09(1 \mathrm{H}, \mathrm{d}, J 7.2, \mathrm{NH}), 12.77(1 \mathrm{H}, \mathrm{br} \mathrm{s}, \mathrm{OH})$ and $\delta_{C}(101$ MHz, d6-DMSO) $17.41\left(\mathrm{CH}_{3 \mathrm{Me}}\right), 31.41(\mathrm{CH}), 55.58\left(\mathrm{CH}_{2}\right), 128.00\left(\mathrm{CH}_{\mathrm{Ph}}\right), 128.75\left(\mathrm{CH}_{\mathrm{Ph}}\right), 132.14$ $\left(\mathrm{CH}_{\mathrm{Ph}}\right), 134.08\left(\mathrm{C}_{\mathrm{Ph}}\right), 167.16\left(\mathrm{CO}_{\mathrm{Ph}}\right), 170.13(\mathrm{COO}), 201.71(\mathrm{COS})$ were in agreement with literature data. ${ }^{14} \mathrm{MS}$ (ESI, positive ion) $\mathrm{m} / \mathrm{z}: 268[\mathrm{M}+\mathrm{H}]^{+}$, ee $>99 \%$,

\section{Supplementary Material}

Biological experiments, ${ }^{1} \mathrm{H}$ and ${ }^{13} \mathrm{C}$ spectra of all reported compounds and development of screening assays are described. 


\section{Acknowledgements}

Justine Simon received a doctoral fellowship from the ARC (NetRBI 2012-2016) of the Belgium French community. A part of this work was financed by the FP6 European Integrated Project EurIntafar (LSHM-CT-2004-512138). We thank Fabrice Bouillenne (CIP, University of Liège, Belgium) for the gift of R39 and André Zapun (IBS, Grenoble, France) for PBP2xR6 and PBP2x5204. We thank Dr. Gabriel Mazzucchelli and co-workers at the GIGA MSL for the HRMS determination.

\section{References}

1. Adam, M.; Damblon, C.; Jamin, M.; Zorzi, W.; Dusart, V.; Galleni, M.; El Kharroubi, A.; Piras, G.; Spratt, B. G.; Keck, W.; Coyette, J.; Ghuysen, J.-M.; Distèche-Nguyen, M.; Frere, J.-M. Biochem. J. 1991, 279, 601-604.

http://dx.doi.org/10.1042/bj2790601

2. Adam, M.; Damblon, C.; Plaitin, B.; Christiaens, L.; Frere, J.-M. Biochem. J. 1990, 270, 525529.

http://dx.doi.org/10.1042/bj2700525

3. Beck, J.; Gharbi, S.; Herteg-Fernea, A.; Vercheval, L.; Bebrone, C.; Lassaux, P.; Zervosen, A.; Marchand-Brynaert, J. Eur. J. Org. Chem. 2009, 85-97

http://dx.doi.org/10.1002/ejoc.200800812.

4. Contreras-Martel, C.; Amoroso, A.; Woon, E. C. Y.; Zervosen, A.; Inglis, S.; Martins, A.; Verlaine, O.; Rydzik, A. M.; Job, V.; Luxen, A.; Joris, B. ; Schofield, C. J.; Dessen, A. ACS Chem. Biol. 2011, 6, 943-951.

http://dx.doi.org/10.1021/cb2001846.

5. Inglis, S. R.; Zervosen, A.; Woon, E. C. Y.; Gerards, T.; Teller, N.; Fischer, D. S.; Luxen, A.; Schofield, C. J. J. Med. Chem. 2009, 52, 6097-6106.

http://dx.doi.org/10.1021/jm9009718.

6. Macheboeuf, P.; Fischer, D. S.; Brown Jr, T.; Zervosen, A.; Luxen, A.; Joris, B.; Dessen, A.; Schofield, C. J. Nat. Chem. Biol. 2007, 3, 565-569.

http://dx.doi.org/10.1038/nchembio.2007.21.

7. Miguet, L.; Zervosen, A.; Gerards, T.; Pasha, F. A.; Luxen, A.; Distèche-Nguyen, M.; Thomas, A. J. Med. Chem. 2009, 52, 5926-5936. http://dx.doi.org/10.1021/jm900625q.

8. Sauvage, E.; Kerff, F.; Terrak, M.; Ayala, J. A.; Charlier, P. FEMS Microbiol. Rev. 2008, 32, 234-258.

http://dx.doi.org/10.1111/j.1574-6976.2008.00105.x.

9. Sauvage, E.; Zervosen, A.; Dive, G.; Herman, R.; Amoroso, A.; Joris, B.; Fonzé, E.; Pratt, R. F.; Luxen, A.; Charlier, P.; Kerff, F. J. Am. Chem. Soc. 2009, 131, 15262-15269. 
http://dx.doi.org/10.1021/ja9051526.

10. Turk, S.; Verlaine, O.; Gerards, T.; Živec, M.; Humljan, J.; Sosič, I.; Amoroso, A.; Zervosen, A.; Luxen, A.; Joris, B.; Gobec, S. PLoS One 2011, 6, e19418. http://dx.doi.org/10.1371/journal.pone.0019418.

11. Woon, E. C. Y.; Zervosen, A.; Sauvage, E.; Simmons, K. J.; Zivec, M.; Inglis, S. R.; Fishwick, C. W. G.; Gobec, S.; Charlier, P.; Luxen, A.; Schofield, C. J. ACS Med. Chem. Lett. 2011, 2, 219-223. http://dx.doi.org/10.1021/ml100260x.

12. Zervosen, A.; Herman, R.; Kerff, F.; Herman, A.; Bouillez, A.; Prati, F.; Pratt, R. F.; Frere, J. M.; Joris, B.; Luxen, A.; Charlier, P.; Sauvage, E. J. Am. Chem. Soc. 2011, 133, 10839-10848. http://dx.doi.org/10.1021/ja200696y.

13. Zervosen, A.; Lu, W. P.; Chen, Z.; White, R. E.; Demuth Jr, T. P.; Frere, J. M. Antimicrob. Agents Chemother. 2004, 48, 961-969. http://dx.doi.org/10.1128/AAC.48.3.961.

14. Llina, A.; Ahmed, N.; Cordaro, M.; Laws, A. P.; Frere, J.-M.; Delmarcelle, M.; Silvaggi, N. R.; Kelly, J. A.; Page, M. I. Biochem. 2005, 7738-7746.

15. Damblon, C.; Zhao, G. H.; Jamin, M.; Ledent, P.; Dubus, A.; Vanhove, M.; Raquet, X.; Christiaens, L.; Frere, J. M. Biochem. J. 1995, 309, 431-436. http://dx.doi.org/10.1042/bj3090431.

16. Greenlee, M. L.; Laub, J. B.; Balkovec, J. M.; Hammond, M. L.; Hammond, G. G.; Pompliano, D. L.; Epstein-Toney, J. H. Bioorg. Med. Chem. Lett. 1999, 9, 2549-2554 http://dx.doi.org/10.1016/S0960-894X(99)00425-4.

17. El-Faham, A.; Albericio, F. Chem. Rev. 2011, 111, 6557-6602. http://dx.doi.org/10.1021/cr100048w.

18. Kricheldorf, H. R.; Kaschig, J. Liebigs Ann. Chem. 1976, 882-890. http://dx.doi.org/10.1002/jlac.197619760511

19. Neises, B.; Steglich, W. Angew. Chem. Int. Ed. 1978, 17, 522-524. http://dx.doi.org/10.1002/anie.197805221.

20. Schwyzer, R.; Hürlimann, C. Helv. Chim. Acta 1954, 37, 155-166. http://dx.doi.org/10.1002/hlca.19540370119.

21. Jamin, M.; Adam, M.; Damblon, C.; Christiaens, L.; Frère, J. M. Biochem. J. 1991, 280, 499506. http://dx.doi.org/10.1042/bj2800499

22. Jamin, M.; Damblon, C.; Millier, S.; Hakenbeck, R.; Frere, J. M. Biochem. J. 1993, 292, 735 741. http://dx.doi.org/10.1042/bj2920735

23. Lakaye, B.; Damblon, C.; Jamin, M.; Galleni, M.; Lepage, S.; Joris, B.; Marchand-Brynaert, J.; Frydrych, C.; Frere, J. M. Biochem. J. 1994, 300, 141-145. http://dx.doi.org/10.1042/bj3000141

24. Stefanova, M.; Bobba, S.; Gutheil, W. G. Anal. Biochem. 2010, 396, 164-166. 
http://dx.doi.org/10.1016/j.ab.2009.09.004.A.

25. Inglis, S. R.; Strieker, M.; Rydzik, A. M.; Dessen, A.; Schofield, C. J. Anal. Biochem. 2012, 420, 41-47. http://dx.doi.org/10.1016/j.ab.2011.08.036.

26. Zervosen, A.; Sauvage, E.; Frère, J.-M.; Charlier, P.; Luxen, A. Molecules 2012, 17, 12478 12505 . http://dx.doi.org/10.3390/molecules171112478.

27. Zervosen, A.; Bouillez, A.; Herman, A.; Amoroso, A.; Joris, B.; Sauvage, E.; Charlier, P.; Luxen, A. Bioorg. Med. Chem. 2012, 20, 3915-3924. http://dx.doi.org/10.1016/j.bmc.2012.04.018.

28. Carapito, R.; Chesnel, L.; Vernet, T.; Zapun, A. J. Biol. Chem. 2006, 281, 1771-1777. http://dx.doi.org/10.1074/jbc.M511506200.

29. Granier, B.; Jamin, M.; Adam, M.; Galleni, M.; Lakaye, B.; Zorzi, W.; Grandchamps, J.; Wilkin, J.; Fraipont, C.; Joris, B.; Duez, C.; Distèche-Nguyen, M.; Coyette, J.; Leyh-Bouille, M.; Dusart, J.; Christiaens, L.; Frère, J.-M.; Ghuysen, J.-M. Methods Enzym. 1994, 244-266, 249.

30. O'Brien, J. L.; Niemann, C. J. Am. Chem. Soc. 1957, 79, 80-85.

http://dx.doi.org/10.1021/ja01558a021. 